\title{
Quadrivalent Inactivated Influenza Vaccine
}

National Cancer Institute

\section{Source}

National Cancer Institute. Quadrivalent Inactivated Influenza Vaccine. NCI Thesaurus.

Code C128455.

A synthetic vaccine consisting of hemagg lutinin ( $\mathrm{HA}$ ) antigens derived from four inactivated influenza viruses, two different influenza type A strains and two different influenza type B strains, with protective immunizing activity. Quadrivalent influenza vaccine (QIV) is formulated annually, based on influenza strains projected to be prevalent in the upcoming flu season. Upon administration, the QIV provides active immunization against the four influenza virus strains, thereby protecting against influenza infection. 